\title{
Propuesta de modelo de mentoría entre iguales en entornos universitarios
}

\author{
Proposal of A Peer Mentoring Model in University Environments \\ Proposta de modelo de mentoria entre iguais no meio universitário
}

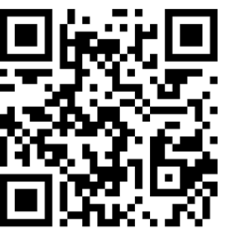

Miguel Aurelio Alonso-García

Universidad Complutense de Madrid Madrid, España

malonsog@ucm.es

http://orcid.org/0000-0003-1584-4436

Recibido • Received • Recebido: 06 / 05 / 2019

Corregido • Revised • Revisado: 03 / 11 / 2020

Aceptado • Accepted $\cdot$ Aprovado: 10 / 12 / 2020

\begin{abstract}
Resumen: A pesar del elevado número de publicaciones sobre mentoría, existen dificultades a la hora de determinar los factores que contribuyen a su éxito. En el presente artículo se realiza una revisión sistemática sobre las variables que se han utilizado como predictoras o antecedentes, las variables de proceso y las de resultados en los estudios de mentoría entre pares en entornos universitarios. La mayor parte de las veces los programas van dirigidos a estudiantes de nuevo ingreso (estudiantado telémaco) a quienes acompañan y facilitan su integración otros grupos de estudiantes de cursos superiores (estudiantado mentor). La revisión permite proponer un modelo sistémico y multinivel que pone en relación los tres tipos de variables y que pretende ser útil para determinar las características que han de tener los programas de mentoría entre iguales en entornos universitarios desde un punto de vista social, organizacional, grupal e individual; las actividades a realizar en las reuniones de mentoría; y las distintas variables en las que puede tener impacto la mentoría como índices de abandono, integración del estudiantado, satisfacción con la carrera, salud, etc.
\end{abstract}

Palabras claves: Mentoría entre iguales; modelo de mentoría; universidad; estudiantes de nuevo ingreso.

\begin{abstract}
In addition to the high number of mentoring publications, there are difficulties determining the factors that contribute to mentoring success. In the present paper, a systematic review is conducted on the variables that have been used as predictors or antecedents, the process variables, and the results of the peer mentoring studies in university environments. Most of the time, the programs are aimed at incoming students (mentees), accompanied by another's students (mentors) of higher levels, who facilitate their integration. The review allows proposing a systemic and multilevel model that relates the three types of variables. It is also intended to be useful to determine the characteristics that peer mentoring programs must have in a university environment from an organizational (group and individual) and social point of view, the activities to be carried out in the mentoring meetings, and the different variables in which mentoring can have an impact such as dropout rates, student integration, career satisfaction, and health.
\end{abstract}

Keywords: Peer mentoring; model of mentoring; university; incoming student. 
http://doi.org/10.15359/ree.25-1.19

http://www.una.ac.cr/educare

educare@una.ac.cr

\begin{abstract}
Resumo: Apesar de termos um elevado número de publicações sobre mentoria, existem muitas dificuldades quando temos de determinar os factores que contribuem ao sucesso da mentoria. No presente artigo realiza-se uma revisão sistemática sobre as variáveis que têm sido utilizadas como preditoras ou antecedentes, as variáveis de proceso e as variáveis de resultados nos estudos de mentoria entre pares no meio universitário. Em muitos casos, os programas são dirigidos a novos alunos ("telêmacos") que são acompanhados por outros alunos do ensino superior (mentores). A revisão permite propor um modelo sistemático e multinível que consegue estabelecer uma relação entre os três tipos de variáveis e que pretende ser útil para determinar as características que os programas de mentoria entre iguais hão de ter no meio universitário, desde um ponto de vista social, organizável, em grupo e individual.As actividades que vão ser realizadas nas reuniões de mentoria; e às diferentes variáveis nas quais a mentoria pode ter impacto, tais como índices de abandono, integração dos estudantes, satisfação com os cursos, saúde, etc.
\end{abstract}

Palavras chave: Mentoria entre iguais; modelo de mentoria; universidade, novos alunos.

\title{
Introducción
}

Los procesos de mentoría se han abordado, fundamentalmente, en los ámbitos organizacional, social y académico y una parte de los estudios se ha centrado en analizar la utilidad de la mentoría.

Los meta-análisis realizados sobre mentoría en entornos organizacionales han abordado los beneficios que obtienen sujetos telémacos (quienes reciben la acción de mentoría) y personas mentoras (persona facilitadora), por ejemplo, Allen et al. (2004) encuentran que la mentoría está más relacionada con los indicadores subjetivos del éxito profesional del estudiantado telémaco (como la satisfacción profesional y laboral), que con indicadores objetivos (como remuneración y promoción); y Ghosh y Reio (2013) analizan los beneficios para la persona mentora entre quien destacan mejoras en satisfacción laboral, compromiso organizacional, desempeño laboral, éxito profesional y reducción en la intención de cambio.

La mentoría informal a jóvenes permite obtener resultados académicos y vocacionales positivos, se hallen en situaciones de exclusión o no, tal y como pone de manifiesto el metaanálisis realizado por van Dam et al. (2018). Aunque algunos estudios (por ejemplo, Rhodes, 2008) encuentran que mientras existen programas que tienen un elevado impacto, otros llegan a tener efectos negativos en los grupos de jóvenes, por lo que señalan que el efecto de la mentoría con jóvenes es moderado.

Las principales revisiones de las publicaciones sobre mentoría en entorno académico las inició Jacobi (1991), incluyendo los estudios publicados con estudiantes de pregrado. Posteriormente, Crisp y Cruz (2009) revisan hasta el año 2007, añadiendo pregrado; los estudios con estudiantado graduado y con estudiantes en el extranjero. Y, por último, Gershenfeld (2014) añade las publicaciones hasta 2012. Ninguno de los tres meta-análisis encuentra evidencias suficientes respecto a la eficacia de los programas de mentoría, a pesar del número elevado de programas que existen. También se han realizado revisiones con objetivos distintos, así Eby et

2

Miguel Aurelio Alonso-García

Los artículos de la Revista Electrónica Educare del Centro de Investigación y Docencia en Educación de la Universidad Nacional, Costa Rica, se comparten bajo términos de la Licencia Creative Commons: Reconocimiento, № Comercial, Sin Obra Derivada 3.0 Costa Rica. Las autorizaciones adicionales a las aquí delimitadas se pueden obtener en el correo: educare@una.cr 
http://doi.org/10.15359/ree.25-1.19

al. (2007) hacen una revisión de bibliográfica para comparar la mentoría académica, laboral y social, y Terrion y Leonard (2010) para determinar las características de una persona mentora en los programas de mentoría entre iguales para tener éxito en su labor.

A pesar del elevado número de publicaciones académicas sobre mentoría (solo en psycinfo aparecen más de 4700 con la palabra clave mentoring), Egege y Kutieleh (2015) señalan la dificultad que existe a la hora de determinar los factores que contribuyen a que tenga éxito. Además, destacan la falta de validez externa de las variables en los estudios ya que las muestras del estudiantado y las cohortes no suelen ser representativas, y que la mayoría de los estudios publicados no describen los métodos utilizados para recopilar y analizar los datos, o no tienen en cuenta otras variables que pueden tener un efecto en los beneficios los programas. En el mismo sentido, Gershenfeld (2014) señala que la proliferación de programas de mentoría no ha estado acompañada por la evidencia empírica sobre su utilidad.

Uno de los problemas es que se utiliza la etiqueta de mentoría para procesos de acompañamiento distintos (Egege y Kutieleh, 2015). En este artículo se hace una propuesta de modelo para mentoría entre iguales en entornos universitarios.

Se propone, como definición de un programa formal de mentoría entre iguales en la universidad, el proceso de acompañamiento en el que el estudiantado de cursos superiores (la persona mentora) mantiene una serie de reuniones con el estudiantado nuevo (telémaco) para facilitarle su integración al entorno y el desarrollo de la carrera, con la supervisión de una persona coordinadora (Alonso García et al., 2012).

El objetivo de este artículo es revisar las variables que se han utilizado como predictoras o antecedentes, las variables de proceso y las de resultados en los estudios de mentoría entre pares en entornos universitarios y proponer un modelo que las relacione.

Para ello se han revisado las publicaciones desde 2012 a la actualidad, utilizando como criterios de búsqueda (en las bases de datos WorldCat, ProQuest Central, Medline, ERIC, y Psycinfo) el término peer mentoring como palabra clave, y combinado con la palabra university en el resumen. Además, se han tenido en cuenta los meta-análisis realizados previamente.

El objetivo de la mentoría dirigida a estudiantes de nuevo ingreso es conseguir su integración, que se sientan de la universidad, que establezcan una red social estable, y que logren resolver la mayor parte de los problemas que tienen que afrontar para tener éxito en la carrera. Un buen indicador de la adaptación al entorno es la permanencia en dichos estudios. De hecho, la revisión de Gershenfeld (2014) encuentra que la teoría que se aplica con mayor frecuencia en los estudios que se realizan con personas mentoras que estudian una carrera en la universidad es la de integración social de Tinto (1993), quien postula que el estudiantado integrado en el entorno del campus, tanto dentro como fuera del aula, tiene más probabilidad de permanecer en la universidad y no abandonarla sin graduarse. 
http://doi.org/10.15359/ree.25-1.19

http://www.una.ac.cr/educare

educare@una.ac.cr

Si se toma como variable criterio o variable resultado de la mentoría la permanencia en la universidad (o abandono) hay distintos modelos que la estudian. Entre ellos Cabrera et al. (2008) destacan el modelo de adaptación (que plantea que las causas del abandono obedecen a una falta de integración académica y social del estudiantado), el modelo psicopedagógico (que busca cómo incrementar las posibilidades de finalizar con éxito los estudios), el modelo estructural (que señala, como variables clave en el abandono el nivel socioeconómico, el trabajo de los padres y madres, el nivel de ingresos en la familia, la situación del mercado de trabajo, etc. ) y el modelo economista (para el cual el abandono es el resultado de la elección entre dedicar tiempo y energía a seguir estudiando, o dejarlo porque percibe mayores beneficios fuera de la universidad). En los distintos modelos se señalan como causas del abandono las características individuales del estudiantado, las características de las carreras, las características de la universidad y el entorno social y familiar.

Además de las mencionadas distintas teorías o modelos que se relacionan con mentoría, como la del capital social, la de intercambio social o la propuesta de modelo orientado a procesos de mentoría de Eby et al. (2013), la teoría del capital social se asocia con el desarrollo de carrera profesional, pues plantea invertir en relaciones sociales con la expectativa de conseguir determinados logros como resultado de la confianza, las expectativas y obligaciones mutuas que los individuos o grupos crean en su entorno (Coleman, 1988).

De acuerdo con la perspectiva del intercambio social, Wang et al. (2010) afirman que es más probable que las personas mentoras realicen un esfuerzo para desarrollar una relación de mentoría y brinden ayuda y apoyo al estudiantado telémaco cuando cree que tales comportamientos serán recíprocos en el futuro o perciban beneficios, incluidos la aprobación y el respeto. Al desarrollar relaciones interpersonales, la confianza comienza en un nivel basado en la cognición, pero si el estudiantado telémaco cree que la persona mentora es competente y de fiar, puede progresar de manera tal que se base en una forma de confianza más alta y más profunda afirmada en el afecto, lo que implica un vínculo emocional cercano.

La propuesta de modelo de Eby et al. (2013) plantea variables de entrada, variables resultado y variables de proceso, y sugieren la necesidad de estudiar aquellas que predicen mejores resultados en un programa de mentoría, analizar cómo mejorar el impacto en las distintas variables criterio, y prestar atención a los procesos.

Tomando como referencia el esquema planteado por Eby et al. (2013) se realiza una propuesta de modelo de mentoría entre iguales en entornos universitarios que incluye variables de entrada para el éxito de la mentoría, variables de proceso que se identifican con el desarrollo del programa (en especial las reuniones entre persona mentora y alumnado telémaco), y variables de resultado. 
http://doi.org/10.15359/ree.25-1.19

\section{Modelo de mentoría entre iguales en entornos universitarios}

\section{Determinantes de la mentoría (input)}

Un modelo debería reflejar las variables que determinan el éxito de un programa de mentoría, algunos estudios lo han tratado de hacer reflejando los resultados de los meta-análisis. Por ejemplo, en ámbito organizacional, Ghosh (2014) resalta aspectos de carácter individual (la proactividad del estudiantado telémaco y su orientación hacia las metas de aprendizaje, y el liderazgo transformacional de la persona mentora), de carácter relacional (la confianza basada en el afecto y la similitud percibida entre la persona mentora y el estudiantado telémaco), y de carácter organizativo (por ejemplo, apoyo organizativo para la mentoría).

En esta propuesta, lo que pasa en las relaciones de mentoría, así como los resultados obtenidos con esta misma, están influidos por las características de la persona mentora y del sujeto telémaco, las características del programa de mentoría, así como el entorno donde se produce el proceso de mentoría, es decir, las características de la facultad y de universidad, y también la situación social y económica. Todos estos aspectos se interrelacionan unos con otros, tal y como aparecen representados en la Figura 1. Se trata de un modelo multinivel, ya que relaciona aspectos de las personas implicadas, las características de los programas, los estudios y la universidad, lo que supone niveles de análisis distintos, pero relacionados entre sí como un sistema con influencias recíprocas.

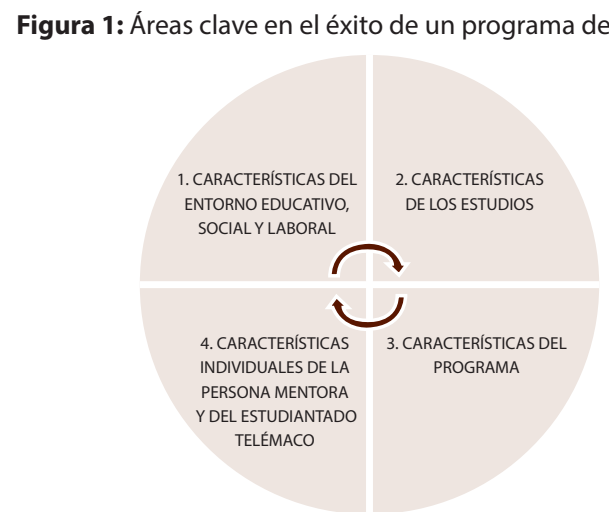

Nota: Elaboración propia.

Cada área contiene distintas variables o antecedentes que pueden ayudar a predecir los resultados que se consigan con un programa de mentoría, a continuación, se abordan cada una de ellas.

\section{a) El entorno educativo, social y laboral}

Las características de la universidad determinan las características de los programas que estas llevan a cabo, que deben reflejar sus valores y sus políticas de actuación, con las adaptaciones pertinentes en función de la facultad o escuela. En los últimos años han aparecido un mayor 
http://doi.org/10.15359/ree.25-1.19

http://www.una.ac.cr/educare

educare@una.ac.cr

número de universidades, lo que ha ocasionado que la nota de admisión a determinadas carreras disminuya significativamente. Sin embargo, otras carreras, como las relacionadas con ciencias de la salud, están viendo un incremento en el número de solicitudes de estudiantes, lo que provoca el efecto contrario, la nota de admisión se incrementa. Indirectamente, mucho estudiantado no logra ser admitido en las carreras que solicitó, acaba cursando carreras que le atraen poco vocacionalmente, lo cual provoca desmotivación y deseos de abandono.

El lugar donde está ubicada la universidad puede dificultar la integración del estudiantado, especialmente cuando se llega de un lugar lejano. No es lo mismo adaptarse a una ciudad pequeña que a una gran ciudad, en la que es más difícil desplazarse, orientarse, o desenvolverse, lo que puede generar indefensión.

Las ofertas de empleo y las condiciones de estas generan expectativas del estudiantado sobre sus posibilidades de trabajar a la hora de tomar la decisión de seguir o no con sus estudios, de acuerdo al citado modelo economista sobre abandono.

\section{b) Características de los estudios}

Las características de los estudios que realiza el estudiantado inciden en el programa de mentoría, ya que en función de la dedicación que implique la carrera y su grado de dificultad, se provocan diferencias en la percepción de presión por parte del estudiantado cuando llega a la universidad. Las creencias sobre la carga o sobrecarga de contenidos, el tiempo disponible para finalizar las tareas y trabajos académicos exigidos, la dificultad de los exámenes muy concentrados en el tiempo, influyen en las estrategias a llevar a cabo por el estudiantado (Cabrera et al., 2008) y, por lo tanto, en el contenido de las reuniones de mentoría.

\section{c) Características del programa de mentoría}

Las características del programa mentoría son relevantes en la consecución de una buena integración de estudiantes de nuevo ingreso, entre ellas se pueden destacar los objetivos del programa, las actividades, las características de las reuniones respecto a número, duración, frecuencia, contenidos..., ya que todo ello garantiza que el programa de mentoría responda a procesos e indicadores que incrementan la calidad.

Algunas de las características de los programas de mentoría formal en la universidad pueden determinar el grado de integración del estudiantado de nuevo ingreso (Alonso García et al., 2012):

- Disponer de una persona coordinadora que se encarga de difundir el programa, seleccionar a las personas mentoras, gestionar su formación, asesorar y organizar el trabajo de las personas mentoras, así como el seguimiento para que desarrollen con eficacia su rol.

6 
http://doi.org/10.15359/ree.25-1.19

- Contar con el reconocimiento explícito de la universidad, que conlleva una serie de exigencias, pero también recompensa con beneficios tangibles a las personas coordinadoras (por ejemplo, pagándoles por la labor o restándoles créditos de su tarea docente) y a las personas mentoras (con el reconocimiento oportuno de créditos), así como asumiendo los costes que conlleva el programa. Un programa de mentoría formal implica que está reconocido por la universidad, lo que incrementa las posibilidades de que se mantenga en el tiempo, ya que, si no está institucionalizado, suele estar vinculado a las personas que los dirigen, que antes o después los dejarán, lo que conlleva con frecuencia la desaparición del programa y sus beneficios.

- Marcar las fechas de inicio y de finalización del programa, es decir, el tiempo desde que se inicia el curso académico hasta que finalizan las reuniones, normalmente con el primer semestre.

- Determinar el número de reuniones a realizar, parece que la satisfacción decrece por debajo de cinco reuniones.

- Recomendar la duración de las reuniones, así la satisfacción del estudiantado telémaco disminuye cuando es menor a 40 minutos.

- Fijar la frecuencia de las reuniones, las dos primeras son más urgentes y se pueden hacer con una semana de intervalo, y después pueden espaciarse más y realizarse cada 15 días hasta la finalización.

- Realizar el proceso de selección de las personas mentoras y determinar sus características, por ejemplo, si hay o no entrevista.

- Definir si el programa es voluntario u obligatorio para el estudiantado telémaco.

- Asegurarse que se imparte la formación oportuna a los agentes clave en el programa: personal coordinador y persona mentora.

- Diseñar y llevar a cabo el proceso y los criterios de asignación de estudiantado telémaco a persona mentora, por ejemplo, en función del género, la edad, el país de procedencia o el turno de clases (Eby et al., 2013; Terrion y Leonard, 2010).

- Determinar el número deseable de estudiantado telémaco por grupo, recomendado entre 5 y 6 por persona mentora.

- Asegurarse de llevar a cabo un buen seguimiento del programa, que permita dar feedback a las personas mentoras de cómo va el proceso. 
http://doi.org/10.15359/ree.25-1.19

http://www.una.ac.cr/educare

educare@una.ac.cr

\section{d) Características de la persona mentora y del estudiantado telémaco}

La persona mentora es uno de los agentes claves para que la mentoría tenga éxito, por ello es esencial realizar una buena selección y también formarla para que adquiera las herramientas y habilidades adecuadas para ejercer adecuadamente su rol. Entre las características a buscar en las personas mentoras se pueden señalar las siguientes:

- Debe estar realizando los mismos estudios que el estudiantado telémaco (Terrion y Leonard, 2010), este requisito debería ser imprescindible: haber pasado por lo mismo para poder ser mentor o mentora.

- Superar el primer curso, lo que supondría llevar al menos un año en la facultad, es la sugerencia señalada por Terrion y Leonard (2007).

- El estudiantado telémaco, al ser de nuevo ingreso, accede al primer curso de la carrera. Pero también es relevante el curso que están realizando las personas mentoras (segundo, tercero, cuarto...) a la hora de la selección. Para Alonso García et al. (2012), si bien es cierto que el estudiantado que cursa segundo tiene más cerca su ingreso y, por lo tanto, les resulta más fácil ponerse en el lugar del estudiantado de nuevo ingreso, las personas mentoras de últimos cursos tienen una visión más amplia de los estudios y la universidad. Sin embargo, no se encuentran diferencias en la satisfacción del estudiantado telémaco en función de dicha variable.

- Decidir si seleccionar como estudiantado mentor a quienes hayan conseguido mayores logros académicos. Mentores y mentoras con buenas notas pueden otorgar credibilidad, pero no está claro, si es mejor modelo para el estudiantado telémaco la persona mentora que más éxito tuvo en el acceso a la universidad, o aquella que ha tenido dificultades y las ha ido solucionando (Terrion y Leonard, 2010).

- Tener experiencia previa como estudiantado mentor puede ser un aspecto a tener en cuenta en la selección (Terrion y Leonard, 2010), siempre y cuando el rendimiento en el año anterior haya sido óptimo y siga teniendo una alta motivación. La organización se beneficia de una persona mentora con experiencia que no hace falta volver a formar.

- Los motivos para ser mentor o mentora parecen ser otra variable clave. Terrion y Leonard (2010) encuentran que tanto las personas mentoras pagadas como las no remuneradas estaban motivadas por razones auto-orientadas, como aprender sobre sí mismas y su satisfacción. No obstante, las personas mentoras pagadas estaban motivadas principalmente por el deseo de ayudar a otras personas, mientras que los compañeros y las compañeras voluntarias informaron que lo hacían para satisfacer necesidades sociales. Además, Terrion y Leonard (2007) encuentran que las personas mentoras que buscan su propio desarrollo quieren mejorar un conjunto de competencias y obtienen mejores resultados. 
- En otros casos se habla de habilidades y competencias a tener en cuenta en la selección de personas mentoras, así aparecen las habilidades de comunicación (Terrion y Leonard, 2007), dimensiones de personalidad como la amabilidad, extraversión y apertura (Goldner, 2016), se relacionan con las expectativas positivas respecto a la mentoría, y si se desarrollan permiten inhibir conductas de ansiedad o conductas negativas por parte del estudiantado telémaco; entusiasmo, interés, energía (Terrion y Leonard, 2007); flexibilidad, que incluye tolerancia hacia la diversidad, hacia posturas distintas, y hacia posibles fracasos (Terrion y Leonard, 2007); y apoyo, ganas de ayudar, palabras de aliento, fomentar que busquen las soluciones (Terrion y Leonard, 2007).

Otro aspecto a tener en cuenta son las características del estudiantado telémaco. Se plantean, como variables importantes de cara a la buena marcha del programa de mentoría, las expectativas que tengan sobre el programa, que están influidas por la información previa antes de inscribirse, y su motivación hacia la carrera y por el programa de mentoría. Además, determinadas características personales: su personalidad, la percepción que tienen sobre la presión de sus padres y madres para que aprueben todo y las dificultades económicas de algunas familias (Cabrera et al., 2008).

El estudiantado telémaco que tiene responsabilidades familiares en el cuidado de las niñas y los niños pequeños, o quienes deben trabajar y estudiar tienen mayores dificultades para compatibilizarlo y más probabilidades de abandonar (Tuero et al., 2018).

\section{La relación de mentoría (el proceso)}

En el apartado anterior se han abordado los antecedentes o variables de entrada que influyen en un programa de mentoría, pero otro aspecto clave es el proceso, es decir, cómo tienen que ser las reuniones de mentoría para que el programa alcance sus objetivos, y dentro de ello se incluyen los aspectos previos y posteriores a las reuniones.

Un programa formal de mentoría entre iguales puede tener éxito en la medida en que personas mentoras y estudiantado telémaco cumplan con las exigencias del mismo programa. Y para que las reuniones sean útiles y la información sea rica es necesario que la persona mentora las prepare previamente, y que consulte la documentación necesaria sobre los temas que va a abordar (Alonso García et al., 2012). Ofrecer información útil forma parte de la función instrumental de la mentoría (Jacobi, 1991) e influye en la confianza basada en la cognición, al facilitar que el sujeto telémaco perciba a la persona mentora como competente (Wang et al., 2010). El apoyo instrumental se ve menos influido por la duración del proceso de mentoría, pero el apoyo emocional y psicológico tiene una mayor incidencia (Eby et al., 2013).

En el desarrollo de las reuniones de mentoría será de mayor calidad e incrementarán las posibilidades de permanencia en el programa en la medida en que la persona mentora lleve a cabo las siguientes actividades: 
http://doi.org/10.15359/ree.25-1.19

http://www.una.ac.cr/educare

educare@una.ac.cr

- Realizar comentarios de comprensión hacia el estudiantado telémaco, y apoyarlo para que se sienta escuchado y adquiera confianza a la hora de resolver los problemas, lo que supone mostrar apoyo, empatía y atención genuina (Terrion y Leonard, 2007), lo que está directamente relacionado con la función de apoyo emocional y psicológico de la mentoría señalada por Jacobi (1991). El apoyo emocional se da cuando en la relación aparece la confianza basada en el afecto, que implica un vínculo emocional cercano (Wang et al., 2010).

- Fomentar la participación activa del estudiantado telémaco en las reuniones para que sean dinámicas y se implique en ellas (Alonso García et al., 2012).

- Orientar en la toma de decisiones, mostrando alternativas de actuación, para que analice las opciones y tome el camino que mejor encaja con su situación.

- Ofrecer feedback sobre sus comentarios y comportamientos, sin juzgarlo, para motivarle e incrementar sus posibilidades de éxito en la carrera (Alonso García et al., 2012).

- Marcar retos y desafíos al estudiantado telémaco que le lleven a la realización de tareas que le permitan desarrollarse (Alonso García et al., 2012).

- Narrar experiencias vividas por la persona mentora para transmitir aprendizajes útiles al estudiantado telémaco, además de mostrar una actitud de igualdad (Terrion y Leonard, 2007), incrementar los resultados académicos (Zevallos y Washburn, 2014), su locus de control interno (Rodger y Tremblay, 2003), y darle pautas para resolver problemas similares, por lo que es útil en la formación de mentores y mentoras (Alonso García et al., 2012).

Posteriormente a las reuniones, es importante analizarlas para buscar estrategias que permitan incrementar la satisfacción y el aprendizaje del estudiantado telémaco, así como su integración en la universidad. Realizar un seguimiento apoyado en técnicas cuantitativas y cualitativas facilita la búsqueda de soluciones a los problemas que surgen (Alonso García et al., 2012).

\section{Los resultados de la mentoría (output)}

Una vez determinadas las variables antecedentes y las de proceso, se abordan las de resultados tomando como referencia la clasificación realizada por Allen y Eby (2007), que divide los resultados obtenidos por la mentoría en seis áreas: comportamientos, actitudes, aspectos relacionados con salud, motivación, relaciones interpersonales y carrera.

\section{a) Comportamientos}

- Integración académica y social del estudiantado (Morales et al., 2016).

- Mejora de las tasas de retención (Jacobi, 1991; Nora y Crisp, 2007). 
http://doi.org/10.15359/ree.25-1.19

- Calificaciones más altas o superar mayor número de asignaturas (Igbo y Sule, 2018; Zevallos y Washburn, 2014).

- Mejora de los conocimientos sobre el entorno, como los recursos y procedimientos universitarios (Chester et al., 2013; Green, 2018).

- Aumento de los comportamientos de las personas mentoras para transmitir conductas académicamente efectivas al estudiantado telémaco (Morales et al., 2016).

- Disminución de conductas poco éticas como robos o agresiones (Allen y Eby, 2007).

\section{b) Actitudes}

- Incremento de la satisfacción con la institución y la satisfacción con la carrera (Allen y Eby, 2007).

- Incremento de la identidad profesional y el orgullo del estudiantado de primer año (Green, 2018), y los valores fundamentales y los principios éticos de la educación superior (Chester et al., 2013).

- Mejora de la confianza y el sentimiento de apoyo (Green, 2018).

\section{c) Relacionadas con la salud}

- Reducción de la ansiedad (Rodger y Tremblay, 2003; Terrion y Leonard, 2007).

- Incremento de la autoeficacia para proseguir una carrera académica (Morales et al., 2016).

\section{d) Motivación}

- Sentido de pertenencia, compromiso y aceptación (Nora y Crisp, 2007).

\section{e) Relaciones interpersonales}

- Calidad de las relaciones del estudiantado con otras personas estudiantes, profesorado, personal, etc. (Chester et al., 2013; Nora y Crisp, 2007).

\section{f) Éxito en la carrera}

Este apartado es menos estudiado, ya que el estudiantad o telémaco no se incorpora al mundo del trabajo hasta años después. En realidad, el éxito en la carrera tendría que ver con la carrera que está cursando y valdrían los indicadores señalados anteriormente. No obstante, pueden señalarse el desarrollo de competencias o habilidades en la persona mentora, que posteriormente pueden 
http://doi.org/10.15359/ree.25-1.19

http://www.una.ac.cr/educare

educare@una.ac.cr

serle útil al acceder al mundo laboral. Entre ellas se pueden señalar las de amabilidad (Goldner, 2016), comunicación y liderazgo (Hogan et al., 2017), flexibilidad y empatía (Terrion y Leonard, 2007), solución de problemas, dirección de equipos, y relaciones interpersonales (Alonso García et al., 2012), o cualquiera de las señaladas como predictoras anteriormente.

La propuesta de modelo de mentoría entre iguales en entornos universitarios, dirigido a estudiantes de nuevo ingreso aparece en la Figura 2, recoge los distintos aspectos planteados hasta el momento.

Figura 2: Modelo de mentoría entre iguales en entorno universitario

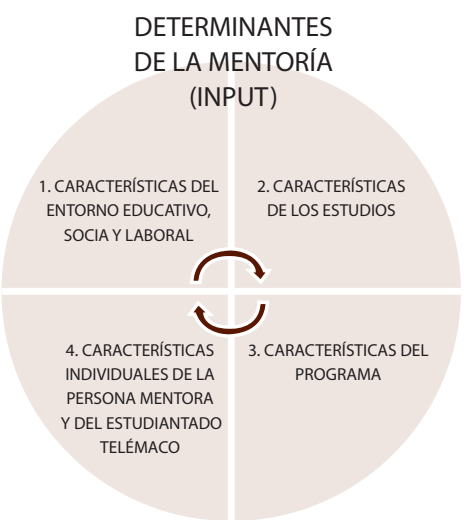

Indicadores de rendimiento
LA RELACIÓN DE MENTORÍA (PROCESO)

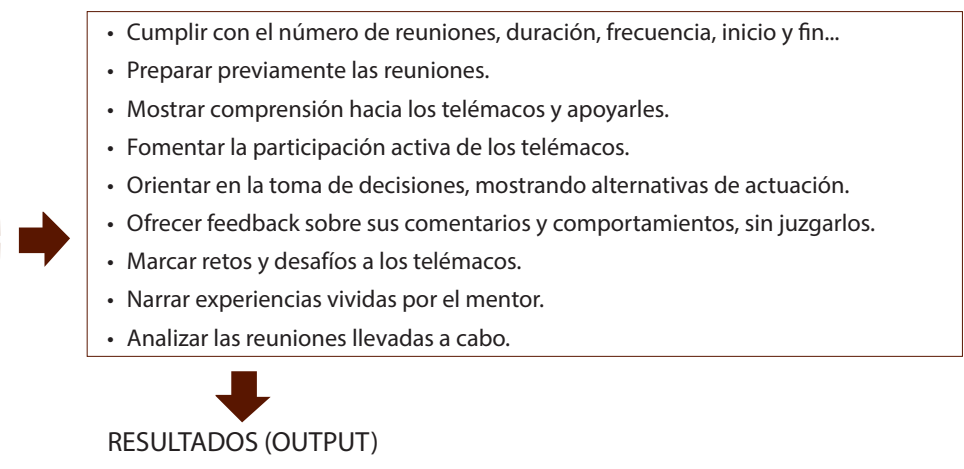

\begin{tabular}{|c|c|c|c|c|}
\hline \multicolumn{2}{|c|}{ Indicadores de rendimiento } & \multicolumn{2}{|c|}{ Acitudes } & Relacionadas con salud \\
\hline \multicolumn{2}{|c|}{$\begin{array}{l}\text { - Tasa de abandono (o la intención de abandonar). } \\
\text { - Conocimientos adquiridos sobre el entorno. } \\
\text { - Comportamientos éticos o no éticos llevados a cabo. } \\
\text { - Calificaciones académicas. }\end{array}$} & \multicolumn{2}{|c|}{$\begin{array}{l}\text { - Satisfacción con el programa de mentoría. } \\
\text { - Satisfacción con la carrera. } \\
\text { - Clima adecuado en la reunión. } \\
\text { - Expectativas. }\end{array}$} & $\begin{array}{l}\text { - Ansiedad. } \\
\text { - Pesimismo. } \\
\text { - Auto-percepción. } \\
\text { - Bienestar psicológico. }\end{array}$ \\
\hline Motivación-Implicación & \multicolumn{2}{|c|}{ Relaciones interpersonales } & \multicolumn{2}{|c|}{ Resultados útiles para los mentores } \\
\hline $\begin{array}{l}\text { - Horas de estudio. } \\
\text { - Compromiso. } \\
\text { - Asistencia a clases. }\end{array}$ & \multicolumn{2}{|c|}{$\begin{array}{l}\text { - Relaciones entre pares. } \\
\text { - Calidad de la relación. } \\
\text { - Asociaciones en las que participa. } \\
\text { - Número de tutorías académicas. }\end{array}$} & \multicolumn{2}{|c|}{$\begin{array}{l}\text { - Desarrollo de habilidades interpersonales. } \\
\text { - Desarrollo de comunicación verbal, amabiliad, } \\
\text { empatía, toma de decisiones, solución de } \\
\text { problemas, dirección de equipos }\end{array}$} \\
\hline
\end{tabular}

Nota: Elaboración propia. 


\section{Discusión}

En el modelo planteado se tratan antecedentes, procesos y resultados; se mencionan como importantes distintas variables de entrada del modelo, relacionándolas con el tipo de comportamientos que lleva la persona mentora en las reuniones (variables de proceso) y por tanto con los resultados que se obtengan. Por ejemplo, respecto a la importancia de estar en una carrera que se ha elegido en primera opción, frente a estar en otra que realmente no se deseaba, en el segundo caso la persona mentora puede poner ejemplos de estudiantes que tampoco la eligieron vocacionalmente y después han acabado disfrutando de los estudios, o sugerirle que finalice el curso y luego tome decisiones sobre si seguir en la misma carrera o cambiar a otra. Este tipo de comentarios en las reuniones suelen tener incidencia positiva en la permanencia de estudiantes, algunos grupos, incluso, abandonan prematuramente la carrera por resultados deficientes en los primeros exámenes (distintos autores y autoras, por ejemplo, Gairín et al., 2014, ponen de manifiesto la relación que hay entre rendimiento académico y permanencia en la universidad; pero, si se quedan, mejoran en los exámenes finales.

En otras ocasiones, las alternativas ofrecidas por la persona mentora en las reuniones, basadas en su experiencia en situaciones similares, permiten (Alonso García et al., 2012):

- Mostrar pautas para facilitarles que superen las asignaturas, por ejemplo: establecer lazos de cooperación con otros compañeros o compañeras a la hora de realizar trabajos de asignaturas; hacer grupos de estudio; descansar; buscar apoyo en manuales específicos, si tiene dificultades para comprender la asignatura; presentarse a actividades voluntarias; acudir a tutorías...

- Integrarse en el entorno a través de la participación en asociaciones, actividades deportivas o culturales, apuntarse a órganos de representación universitaria...

- Hacer un buen uso de los recursos, ya que la persona mentora ofrece información, y ayuda en relación con servicios como la biblioteca, recursos informáticos, rectorado, secretaría, matrícula, créditos, laboratorios, medios de transporte...

Cumplir con ciertas buenas prácticas en el desarrollo de los programas de mentoría, es clave para el éxito $y$, cuando no se cumplen, lo habitual es que no funcionen adecuadamente y no se mantengan en el tiempo (ausencia de formación de personas coordinadoras y mentoras, falta de seguimiento de las reuniones, programas con pocas reuniones, insuficiente duración o poca frecuencia, etc.). Muchas de estas buenas prácticas están desarrolladas en publicaciones previas (Alonso García et al., 2012); sin embargo, hay otros aspectos que son susceptibles de 
http://doi.org/10.15359/ree.25-1.19

http://www.una.ac.cr/educare

educare@una.ac.cr

mejora, por ejemplo, la asignación de estudiantado telémaco a mentores o mentores o reducir su abandono de los programas.

Es importante que se aborden las distintas características del programa. En este sentido, pensamos que las variables de entrada y de proceso mencionadas en el modelo ayudarán a este cometido, lo que facilitará, posteriormente, realizar comparaciones entre investigaciones distintas. Dichas variables también pueden utilizarse como variables predictoras para determinar aquellas que más peso tienen en la eficacia de los programas de mentoría. Conviene plantear un análisis multinivel que considere los datos de carácter individual, los datos de los grupos de mentoría (la persona mentora con su estudiantado telémaco) y los de cada centro de estudios, para así conocer las problemáticas con mayor profundidad y encontrar las soluciones más eficaces.

Es probable que, como ocurre en entorno organizacional (Allen et al., 2004), la mentoría entre iguales tenga más influencia en resultados de tipo subjetivo, como la satisfacción con la carrera, que con otros de tipo objetivo como notas. Pero es necesario hacer más esfuerzos para buscar indicadores de rendimiento (notas, tasa de abandono, conocimientos del entorno...), y salud, entre otros. Además, es necesario utilizar diseños cuasiexperimentales que permitan el contraste, preferiblemente, con la presencia de grupo de control con similares características.

Es difícilmente justificable que el elevado número de publicaciones académicas sobre mentoría no vaya acompañado del rigor necesario en los diseños, no haya acuerdo en la definición de conceptos y siga habiendo confusión entre tutoría, mentoría, mentoría entre iguales, o programas de acompañamiento (Egege y Kutieleh, 2015). Todo ello complica en exceso el avance en el ámbito de la mentoría académica y dificulta la realización de estudios sistemáticos sobre aspectos concretos.

Dentro de las limitaciones de este estudio, señalar que es una primera propuesta de modelo, elaborada a raíz de la revisión bibliográfica, pero que habrá que validarlo de forma empírica y contrastarlo con la comunidad académica.

La creación de sellos de calidad de la mentoría puede contribuir a fijar estándares que mejoren la calidad de los programas y, como consecuencia, los resultados obtenidos con estos mismos.

\section{Declaración de Material complementario}

Este artículo tiene disponible, como material complementario:

-La versión preprint del artículo en https://doi.org/10.5281/zenodo.3534617 


\section{Referencias}

Allen, T. D. y Eby, L. T. (2007). Common bonds: An integrative view of mentoring relationships. En T. D. Allen y L. T. Eby (Eds.), The blackwell handbook of mentoring: A multiple perspectives approach (pp. 397-419). Blackwell Publishing. https://doi.org/10.1111/ b.9781405133739.2007.00024.x

Allen, T. D., Eby, L. T., Poteet, M. L., Lentz, E. y Lima, L. (2004). Career benefits associated with mentoring for proteges: A meta-analysis. Journal of Applied Psychology, 89(1), 127-136. https://doi.org/10.1037/0021-9010.89.1.127

Alonso García, M. A., Calles Doñate, A. y Sánchez Ávila, C. (2012). Diseño y desarrollo de programas de mentoring en organizaciones. Síntesis.

Cabrera, L., Bethencourt, J. T., Álvarez Pérez, P. y González Alfonso, M. (2008). El problema del abandono de los estudios universitarios. RELIEVE, 12(2), 171-203. https://doi.org/10.7203/ relieve.12.2.4226

Chester, A., Burton, L. J., Xenos, S. y Elgar, K. (2013). Peer mentoring: Supporting successful transition for first year undergraduate psychology students. Australian Journal of Psychology, 65(1), 30-37. https://doi.org/10.1111/ajpy.12006

Coleman, J. S. (1988). Social capital in the creation of human capital. American Journal of Sociology, 94, S95-S120. https://doi.org/10.1086/228943

Crisp, G. y Cruz, I. (2009). Mentoring college students: A critical review of the literature between 1990 and 2007. Research in Higher Education, 50(6), 525-545. https://doi.org/10.1007/ s11162-009-9130-2

Eby, L., Allen, T., Hoffman, B. J., Baranik, L. E., Sauer, J. B., Baldwin, S., Morrison, M. A., Kinkade, K. M., Maher, C. P., Curtis, S. y Evans, S. C. (2013). An interdisciplinary meta-analysis of the potential antecedents, correlates, and consequences of protégé perceptions of mentoring. Psychological Bulletin, 139(2), 441-476. https://doi.org/10.1037/a0029279

Eby, L. T., Rhodes, J. E. y Allen, T. D. (2007). Definition and evolution of mentoring. En T. D. Allen y L. T. Eby (Eds.), The Blackwell handbook of mentoring: A multiple perspectives approach (pp. 7-20). Blackwell Publishing. https://doi.org/10.1002/9780470691960.ch2 
http://doi.org/10.15359/ree.25-1.19

http://www.una.ac.cr/educare

educare@una.ac.cr

Egege, S. y Kutieleh, S. (2015). Peer mentors as a transition strategy at university: Why mentoring needs to have boundaries. Australian Journal of Education, 59(3), 265-277. https://doi. org/10.1177/0004944115604697

Gairín, J., Triado, X. M., Feixas, M., Figuera, P., Aparicio-Chueca, P. y Torrado, M. (2014). Student dropout rates in Catalan universities: Profile and motives for disengagement. Quality in Higher Education, 20(2), 165-182. https://doi.org/10.1080/13538322.2014.925230

Gershenfeld, S. (2014). A review of undergraduate mentoring programs. Review of Educational Research, 84(3), 365-391. https://doi.org/10.3102/0034654313520512

Ghosh, R. (2014). Antecedents of mentoring support: A meta-analysis of individual, relational, and structural or organizational factors. Journal of Vocational Behavior, 84(3), 367-384. https://doi.org/10.1016/j.jvb.2014.02.009

Ghosh, R. y Reio, T. G. Jr. (2013). Career benefits associated with mentoring for mentors: A meta-analysis. Journal of Vocational Behavior, 83(1), 106-116. https://doi.org/10.1016/j. jvb.2013.03.011

Goldner, L. (2016). Protégés' personality traits, expectations, the quality of the mentoring relationship and adjustment: A big five analysis. Child \& Youth Care Forum, 45(1), 85-105. https://doi.org/10.1007/s10566-015-9319-9

Green, J. L. (2018). Peer support systems and professional identity of student nurses undertaking a uk learning disability nursing programme. Nurse Education in Practice, 30, 56-61. https:// doi.org/10.1016/j.nepr.2017.11.009

Hogan, R., Fox, D. y Barratt-See, G. (2017). Peer to peer mentoring: Outcomes of third-year midwifery students mentoring first-year students. Women and Birth, 30(3), 206-213. https://doi.org/10.1016/j.wombi.2017.03.004

Igbo, I. N.y Sule, E. N. (2018). Peer mentoring as a successful retention strategy for a baccalaureate nursing program in a historically black college and university. Nursing Education Perspectives, 40(3), 192-193. https://doi.org/10.1097/01.NEP.0000000000000346

Jacobi, M. (1991). Mentoring and undergraduate academic success: A literature review. Review of Educational Research, 61(4), 505-532. https://doi.org/10.3102/00346543061004505

Morales, E. E., Ambrose-Roman, S. y Perez-Maldonado, R. (2016). Transmitting success: Comprehensive peer mentoring for at-risk students in developmental math. Innovative Higher Education, 41(2), 121-135. https://doi.org/10.1007/s10755-015-9335-6 
Nora, A. y Crisp, G. (2007). Mentoring students: Conceptualizing and validating the multi-dimensions of a support system. Journal of College Student Retention: Research, Theory \& Practice, 9(3), 337356. https://doi.org/10.2190/CS.9.3.e

Rhodes, J. E. (2008). Improving youth mentoring through research-based practice. American Journal of Community Psychology, 41(1-2), 35-42. https://doi.org/10.1007/s10464-007-9153-9

Rodger, S. y Tremblay, P. F. (2003). The effects of a peer mentoring program on academic success among first year university students. Canadian Journal of Higher Education, 33(3), 1-17. https:// eric.ed.gov/?id=EJ788475

Terrion, J. L. y Leonard, D. (2007). A taxonomy of the characteristics of student peer mentors in higher education: Findings from a literature review. Mentoring \& Tutoring; Partnership in Learning, 15(2), 149-164. https://doi.org/10.1080/13611260601086311

Terrion, J. L. y Leonard, D. (2010). Motivation of paid peer mentors and unpaid peer helpers in higher education. International Journal of Evidence Based Coaching and Mentoring, 8(1), 85103. https://radar.brookes.ac.uk/radar/items/8c6a37ad-0979-4c02-95f5-80f8071f62e6/1/

Tinto, V. (1993). Leaving college: Rethinking the causes and cures of student attrition. University of Chicago Press. https://doi.org/10.7208/chicago/9780226922461.001.0001

Tuero, E., Cervero, A., Esteban, M. y Bernardo, A. (2018). ¿Por qué abandonan los estudios universitarios? Variables de influencia en el planteamiento y consolidación del abandono. Educación XX1, 21(2), 131-154. https://doi.org/10.5944/educxx1.20066

van Dam, L., Smit, D., Wildschut, B., Branje, S. J. T., Rhodes, J. E., Assink, M. y Stams, G. J. J. M. (2018). Does natural mentoring matter? A multilevel meta-analysis on the association between natural mentoring and youth outcomes. American Journal of Community Psychology, 62(1-2), 203-220. https://doi.org/10.1002/ajcp.12248

Wang, S., Tomlinson, E. C. y Noe, R. A. (2010). The role of mentor trust and protégé internal locus of control in formal mentoring relationships. Journal of Applied Psychology, 95(2), 358-367. https://doi.org/10.1037/a0017663

Zevallos, A. L. y Washburn, M. (2014). Creating a culture of student success: The SEEK scholars peer mentoring program. About Campus, 18(6), 25-29. https://doi.org/10.1002/abc.21141 